\title{
Pericyclic Reactions in Ether Biofuels
}

\author{
Juan-Carlos Lizardo-Huerta, Baptiste Sirjean, Pierre-Alexandre Glaude, René Fournet
}

Laboratoire Réactions et Génie des Procédés, CNRS, Université de Lorraine, ENSIC, 1 rue Grandville BP 2045154001 Nancy Cedex, France

\begin{abstract}
Unimolecular decompositions of acyclic ethers through a pericyclic mechanism, i.e., alcohol elimination, have been shown to play a crucial role in the high-temperature combustion of these compounds. The production of new fuels derived from biomass has led to a renewed interest into the combustion chemistry of ethers. A large fraction of ethers produced as potential biofuels features a cyclic structure. The pericyclic reactions in these cyclic structures, with or without a lateral alkyl group, remains unknown. In this work, we performed a systematic theoretical study on the pericyclic reactions in acyclic and cyclic ethers. Envisaged concerted reactions includes the classical alcohol formation and a $\mathrm{H}_{2}$ eliminations that was recently shown to play a non negligible role in the thermal decomposition of tetrahydrofuran [Proc. Combust. Inst., 35 (2015) 533]. Theoretical calculation performed in this work demonstrated that $\mathrm{H}_{2}$ elimination in acyclic ethers is negligible. In the case of cyclic ethers (tetrahydrofuran and tetrahydropyran), the branching ratio of the unimolecular pericyclic reaction strongly depends on the presence of a lateral alkyl group bonded to the carbon atom in position 2. If an alkyl group is present, the alcohol formation is favored through an exo 4-center rearrangement, that we newly defined in this work. If no lateral alkyl group is available in position 2, endo alcohol formation and $\mathrm{H}_{2}$ eliminations are equivalently important. Reaction rate rules were established to include pericyclic decomposition reactions in detailed chemical kinetic models of ether combustion.
\end{abstract}

\section{Keywords:}

theoretical kinetics; quantum chemistry; biofuel ; ethers; 


\section{Introduction}

Ethers have attracted a great interest as promising transportation fuels produced from biomass [1]. Ether compounds that can be used as additives or biofuels can be divided into two categories: acyclic and cyclic ethers. Acyclic ethers, dimethyl ether $\left(\mathrm{DME}:\left(\mathrm{CH}_{3}\right)_{2} \mathrm{O}\right)$ and its derivatives, are wellknown octane improvers produced from alcohols. Cyclic ethers, either saturated or unsaturated furans, are mostly produced from catalytic treatments of lignocellulosic biomass. The potential of these compounds have led to a large number of studies aiming to unravel their combustion chemistry at the molecular level. Tran et al. [2] listed all the detailed kinetic models available for these compounds in their 2012 review. Since then, a kinetic mechanism on dibutyl ether $\left(\mathrm{DBE}:\left(\mathrm{C}_{4} \mathrm{H}_{9}\right)_{2} \mathrm{O}\right)$ has been published [3], and new versions of published combustion kinetic models of ethers are proposed regularly in the literature [4-6]. Those studies showed that unimolecular eliminations are crucial in the high temperature combustion chemistry of acyclic ethers (larger than DME).

Danby and Freeman first detected ethanol as a product of diethyl ether $\left(\mathrm{DEE}:\left(\mathrm{C}_{2} \mathrm{H}_{5}\right)_{2} \mathrm{O}\right)$ thermal decomposition in 1958 [7], and ascribed its formation to the reaction

$$
\mathrm{C}_{4} \mathrm{H}_{10} \mathrm{O} \rightarrow \mathrm{C}_{2} \mathrm{H}_{5} \mathrm{OH}+\mathrm{C}_{2} \mathrm{H}_{4}
$$

where the mechanism of formation of ethylene and ethanol was unknown but probably first order. In 1963, Laidler and McKenney proved that the formation of the products of reaction (1) occurred from a molecular elimination in DEE [8] and subsequently proposed a rate constant with an activation energy of $83.8 \mathrm{kcal} \mathrm{mol}^{-1}$ and a frequency factor of $2.75 \times 10^{18} \mathrm{~s}^{-1}$. Fifteen years later, Foucaut and Martin [9] measured a rate constant of $1.0 \times 10^{13}\left(\mathrm{~s}^{-1}\right) \exp \left(-62.2\left(\mathrm{kcal} \mathrm{mol}^{-1}\right) / \mathrm{RT}\right)$ between 763 and $798 \mathrm{~K}$. Large discrepancies can be observed between these rate parameters and those proposed by Laidler and McKenney. A comparison of the two rate constants at $800 \mathrm{~K}$, where the experimental temperature bounds are the closest, ultimately leads to a factor of 2.9 (Foucaut and Martin's rate is higher than Laidler and McKenney). In 2010, Yasunaga et al. [10] studied the pyrolysis of ethyl tert-butyl ether 
(ETBE: $\left.\mathrm{C}_{2} \mathrm{H}_{5}-\mathrm{O}-\mathrm{C}\left(\mathrm{CH}_{3}\right)_{3}\right)$ behind reflected shock waves and showed that the 4-center elimination of alcohol is dominant in the initial decomposition steps of ETBE. They calculated the high-pressure rate constant for this pericyclic reaction, using quantum chemistry, at the MP4/cc-pVTZ//MP2/cc-pVTZ level of theory. It can be noted that no treatment of internal rotors was reported by the authors. The computed kinetic parameters of this reaction were subsequently used for reaction (1) in an experimental and modeling study on DEE pyrolysis and combustion [11]. Their computed rate constant was reported to be approximately three times higher than that of Foucaut and Martin. Very recently, Al Rashidi et al. [12] calculated the high-pressure limit rate constant for the 4-center pericyclic elimination of butanol in dibutylether using CBS-QB3 and G4 level of theory. Internal rotations were treated using the approximation of Pitzer and Gwinn [13], based on relaxed scans performed at the B3LYP/6-31+G(d,p) level of theory. The lowest critical energy computed by these authors is $67.7 \mathrm{kcal} \mathrm{mol}^{-1}$ (G4 at $0 \mathrm{~K}$ ) which is $7.7 \mathrm{kcal} \mathrm{mol}^{-1}$ higher than the one calculated by Yasunaga et al. (MP4/cc-pVTZ//MP2/cc-pVTZ at $0 \mathrm{~K})$. The total high-pressure limit rate constant calculated by $\mathrm{Al}$ Rashidi et al. at $800 \mathrm{~K}$ is $3.56 \times 10^{-6} \mathrm{~s}^{-1}$, which is lower by a factor of 28 compared to the value reported by Foucaut and Martin.

The role of the pericyclic elimination of alcohol in cyclic ether has almost not been studied in the literature. In the case of unsaturated cyclic ethers, i.e., furan derivatives, this kind of elimination seems not to be possible. In our work on 2,5-dimethylfuran (2,5-DMTHF), no 4-center elimination transition state was optimized at the B3LYP/cbsb7 level of theory despite our attempts [14]. In the case of saturated cyclic ethers, three studies can be mentioned. Moshammer et al. [15] proposed a detailed chemical kinetic model to simulate the combustion of 2-methyltetrahydrofuran (2-MTHF) in flames. In their model, eliminations reaction from the fuel are included, leading to the formation of formaldehyde and butene, acetaldehyde and propene or acetone and ethylene. No detail was given for the determination of these reactions and their associated kinetic parameters. Verdicchio et al. [16] 
theoretically explored the unimolecular decomposition of tetrahydrofuran (THF) and envisaged a molecular elimination, similar to the one presented in reaction (1)

$$
\text { tetrahydrofuran } \rightarrow \mathrm{CH}_{2} \mathrm{CHCH}_{2} \mathrm{CH}_{2} \mathrm{OH}
$$

where the cyclic structure of THF leads to the formation of but-3-en-1-ol, an unsaturated alcohol. This elementary step involves a bicyclic transition state structure, where the $\mathrm{H}$-atom bonded to the carbon 3 is transferred on the oxygen atom. A computed energy barrier of $80.4 \mathrm{kcal} \mathrm{mol}^{-1}$ was reported by the authors for reaction (2) at the CBS-QB3 level of theory. Verdicchio et al. also reported an original $\mathrm{H}_{2}$ elimination reaction in THF leading to a zwitterionic $\mathrm{C}_{4} \mathrm{H}_{6} \mathrm{O}$ cyclic species that further isomerizes to 2,3-dihydrofuran. It was shown, in this study, that the $\mathrm{H}_{2}$ elimination is competitive with the pericyclic rearrangement yielding the unsaturated alcohol. Tran et al. [17] proposed a combustion and pyrolysis kinetic model for tetrahydropyran (THP) that included unimolecular decomposition reactions of the fuel based on analogies with the work of Verdicchio et al. [16]. They calculated a high-pressure limit rate constant at the CBS-QB3 level of calculation (including 1-DHR treatment of internal rotors), and reported a computed rate constant of $4.20 \times 10^{5} \mathrm{~T}^{2.6} \exp \left(-73.3\left(\mathrm{kcal} \mathrm{mol}^{-1}\right) / \mathrm{RT}\right) \mathrm{s}^{-1}$. The fitted activation energy of $73.3 \mathrm{kcal} \mathrm{mol}^{-1}$ is lower than the similar pericyclic reaction in THF by $7 \mathrm{kcal} \mathrm{mol}^{-1}$. These authors did not envisaged the $\mathrm{H}_{2}$ elimination reaction in their model. It can be noted that no pericyclic reaction is included in the kinetic models of THP combustion proposed by Labbe et al. [18] and that Dagaut et al. [19] considered lumped molecular elimination in their mechanism, based on experiments performed on THF.

The present study aims at unraveling the role of pericyclic reactions in ethers combustion. In particular, the influence of a lateral alkyl chain on a cyclic ether will be investigated as well as the size of the cyclic structure. The role of $\mathrm{H}_{2}$ eliminations in ethers will also be clarified. Reaction rate rules will be determined that will be useful in the development of ether combustion kinetic models. 


\section{Computational details}

Potential energy surfaces were calculated using different approaches. For the smaller compounds (less than 6 heavy atoms), geometry optimizations and frequency calculations were performed at the M06-2X/6-311+G(2d,p) level of theory. Single point energy calculations were computed using the CCSD(T)-F12 approach with the VDZ-F12 basis set (noted CCSD(T)-F12//M06-2X). Frequencies obtained at the M06-2X level of calculation were scaled using a factor of 0.983 [20]. For compounds featuring more than 6 heavy atoms, the CCSD(T)-F12 method was found to be too heavy to compute electronic energies within a reasonable CPU time. Instead, we performed a benchmark on small compounds electronic energies using $\operatorname{CCSD}(\mathrm{T})-\mathrm{F} 12$ results as a reference and determined the most efficient method to study this system. Results of this benchmark, given in Table S1, show that the G4 composite method is the best choice in this case. Therefore, the electronic structures of heavier compounds were computed at this level of theory. T1 diagnostics were systematically calculated and no multi-reference issue has been found in stable and saddle points structures. Analysis of vibrational frequencies, obtained from both $\mathrm{G} 4$ and $\operatorname{CCSD}(\mathrm{T})-\mathrm{F} 12 / / \mathrm{M} 06-2 \mathrm{X}$ results, confirmed that all transition structures have one imaginary frequency. High-pressure limit rate constants were calculated using THERMROT [21], an in-house code that allows a 1-DHR treatment of internal rotations and takes into account the coupling of torsional modes using the method proposed by Vansteenkiste et al. [22] . Quantum tunneling was considered using the Eckart approach for reactions involving H-atom transfer [23]. 1-DHR treatment was used with torsional potential calculated from relaxed scans. For cyclic transition states, the vibrational modes of the cyclic part of the molecular structure were described within the HO approximation, while lateral groups were treated as hindered rotors. 


\section{Results and discussion}

\subsection{Pericyclic reactions of acyclic ethers}

The simplest acyclic ether is DME and its unimolecular decomposition reactions have been studied in great details [24]. The initial C-O bond fission $\mathrm{CH}_{3} \mathrm{OCH} 3 \rightarrow \mathrm{CH}_{3} \bullet+\mathrm{CH}_{3} \mathrm{O} \bullet$ has been thought to be the sole unimolecular initiation mechanism for decades as the size of DME does not allow a 4-center pericyclic elimination to occur. Recently, Sivaramakrishnan et al. [24] showed that a roaming pathway $\mathrm{CH}_{3} \mathrm{OCH} 3 \rightarrow \mathrm{CH}_{4}+\mathrm{CH}_{2} \mathrm{O}$ can also play a non negligible role in the unimolecular decomposition of DME. In their study, $\mathrm{H}_{2}$ elimination from DME depicted in Figure 1, was not considered.

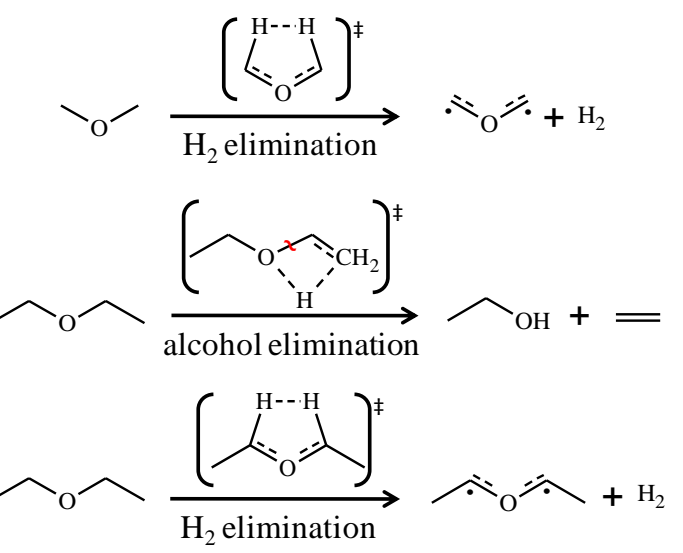

Figure 1: Pericyclic reactions of DME and DEE.

Our calculations predict that the elimination of $\mathrm{H}_{2}$ from DME faces a critical energy of $79.2 \mathrm{kcal}$ $\mathrm{mol}^{-1}(\mathrm{CCSD}(\mathrm{T})-\mathrm{F} 12 / / \mathrm{M} 06-2 \mathrm{X}$ result at $0 \mathrm{~K})$. Sivaramakrishnan et al. [24] computed a detailed PES for DME unimolecular decomposition at the CCSD(T)/CBS//CCSD(T)/aug-cc-pvdz level of theory. At 0 K, they computed a C-O bond dissociation energy of $82.5 \mathrm{kcal} \mathrm{mol}^{-1}$ and, among the other pathways explored, they proposed a 3-center alcohol elimination $\mathrm{CH}_{3} \mathrm{OCH}_{3} \rightarrow \mathrm{CH}_{2}+\mathrm{CH}_{3} \mathrm{OH}$ that represents the lowest energy - tight - saddle point of the surface $\left(83.8 \mathrm{kcal} \mathrm{mol}^{-1}\right)$. On the other hand, the roaming saddle point determined by these authors lies $80.5 \mathrm{kcal} \mathrm{mol}^{-1}$ above DME. $\mathrm{H}_{2}$-elimination transition state 
energy calculated in our work is found to lie $3.3 \mathrm{kcal} \mathrm{mol}^{-1}$ below the asymptotic $\mathrm{CH}_{3} \bullet+\mathrm{CH}_{3} \mathrm{O} \bullet$ dissociative limit and $1.3 \mathrm{kcal} \mathrm{mol}^{-1}$ below the roaming saddle point of the Sivaramakrishnan et al. [24] study. A difference of $3.3 \mathrm{kcal} \mathrm{mol}^{-1}$ in the activation energy leads to a factor of 5 at $1000 \mathrm{~K}$ between the rate constants of initiation and elimination. However pre-exponential factors for initial bond fissions are known to be high compared to tight transition states such as the one involved in $\mathrm{H}_{2}$ elimination. Figure 2 depicts the high-pressure limit rate constants for $\mathrm{H}_{2}$ elimination and initial $\mathrm{C}-\mathrm{O}$ bond fission in DME (from Sivaramakrishnan et al.).

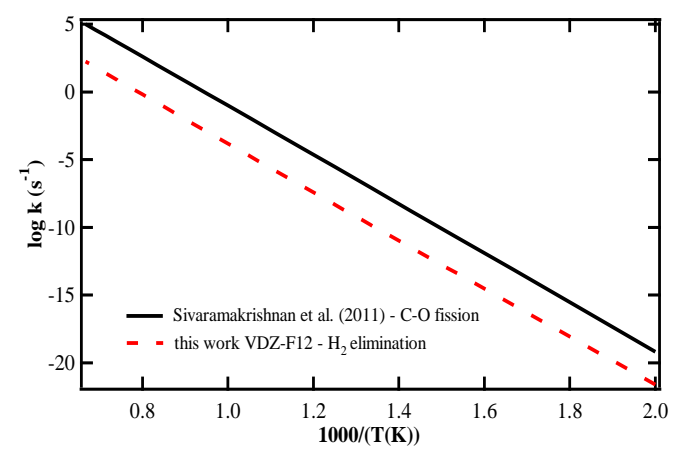

Figure 2: Comparison of high-pressure limit rate constants for $\mathrm{H}_{2}$-elimination (this work) and $\mathrm{C}$-O bond fission in DME [24].

It can be seen in Figure 2 that despite the low energy barrier of $\mathrm{H}_{2}$ elimination, entropic effects favor the initial bond-fission by about two orders of magnitude (a factor of 650 at $1000 \mathrm{~K}$ ). Influence of pressure was probed using master equation (ME) modeling with the PAPER code [25-26]. Details of the parameters used in the resolution of the ME can be found in Table S2. At 0.01 bar and $1000 \mathrm{~K}$, the pressure dependent rate constant of DME initial C-O bond fission (taken from ref. 23) is 60 times larger than the $\mathrm{H}_{2}$ elimination rate coefficient. The concerted elimination of hydrogen is therefore expected to be negligible (less than $2 \%$ of the branching ratio) compared to initial $\mathrm{C}-\mathrm{O}$ bond fission even at lower pressures.

Figure 3 presents the high-pressure limit rate constants for $\mathrm{C}-\mathrm{O}$ bond fission, $\mathrm{H}_{2}$ elimination and alcohol elimination in DEE. The size of DEE allows the occurring of the 4-center alcohol elimination. 


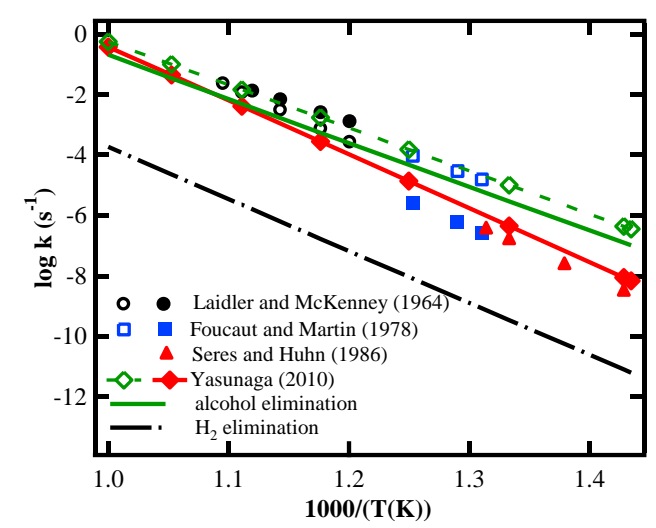

Figure 3: Comparison of high-pressure limit rate constants for $\mathrm{H}_{2}$-elimination, alcohol elimination and $\mathrm{C}$-O bond fission in DEE. Lines represent rate constants calculated in this work. Open symbols are experimental values for the alcohol elimination, and filled symbols represent experimental kinetic parameters for the initial $\mathrm{C}-\mathrm{O}$ bond fission.

From Figure 3, it can be seen that the 4-center pericyclic elimination of alcohol is competitive with the initial $\mathrm{C}-\mathrm{O}$ bond fission. A disagreement can be observed between the measured unimolecular initiation in DEE. Foucaut and Martin [9] and Seres and Huhn [27] experiments are in agreement, while Laidler and McKenney [8] values are higher. It can be noted that all experimental measurements were performed bellow atmospheric pressure: Foucault and Martin (50 - 100 Torr), Laidler and McKenney (30 - 360 Torr) and Seres and Huhn (75 - 300 Torr). Yasunaga et al. estimated the high pressure limit rate constant for the initial $\mathrm{C}-\mathrm{O}$ bond fission in $\mathrm{DEE}$ and found values slightly higher than those measured by Foucaut and Martin and Seres and Huhn, 6 and 2 times faster at 800 K, respectively. Still, the current state-of-the-art in the literature is that alcohol elimination is dominant over unimolecular initiation below $1000 \mathrm{~K}$, and competitive at higher temperatures. For this 4-centers pericyclic reaction, CCSD(T)-F12//M06-2X calculations yield an energy barrier of $67.3 \mathrm{kcal} \mathrm{mol}^{-1}$, in agreement with the value of $67.7 \mathrm{kcal} \mathrm{mol}^{-1}$ computed by Al Rashidi et al. [12]. Our computed high-pressure limit rate constant is in good agreement with experimental data (a maximum absolute deviation of $70 \%$ ). The elimination of hydrogen faces an energy barrier of $77.3 \mathrm{kcal} \mathrm{mol}^{-1}$ that is $10 \mathrm{kcal} \mathrm{mol}^{-1}$ above the value computed for alcohol elimination. Again, $\mathrm{H}_{2}$ elimination can be neglected in DEE thermal decomposition pathways. This stands as a general conclusion for acyclic ethers, where the 4-center 
alcohol elimination and the initial $\mathrm{C}-\mathrm{O}$ bond fission are dominant. The role of roaming reaction has yet to be clarified as disagreements between theory and experiments have been reported: Sivaramakrishnan et al. [23] observed an experimental branching ratio of 0.19 for the roaming mechanism while a branching ratio of 0.02 was computed using theoretical calculations. For the reaction of alcohol elimination in acyclic ethers, we recommend high pressure limit rate constants that depends on the nature of the transferred $\mathrm{H}$-atom presented in Table 1.

Table 1: Reaction rate rule for alcohol elimination in acyclic ether.

\begin{tabular}{cccc} 
H-type & \multicolumn{3}{c}{$\mathrm{k}=\mathrm{AT}^{\mathrm{n}} \exp (-\mathrm{E} / \mathrm{RT})$} \\
\cline { 2 - 4 } & $\mathrm{A}\left(\mathrm{s}^{-1}\right)$ & $\mathrm{n}$ & $\mathrm{E}(\mathrm{cal})$ \\
\hline Primary & $5.727 \times 10^{4}$ & 2.612 & 61921.3 \\
Secondary & $1.397 \times 10^{5}$ & 2.445 & 62830.3 \\
Tertiary & $1.559 \times 10^{6}$ & 2.132 & 64297.8 \\
\hline \multicolumn{3}{c}{ * No additional correction for reaction path degeneracy is required }
\end{tabular}

\subsection{Pericyclic reactions of cyclic ethers}

The role of pericyclic reactions in cyclic ether thermal degradation has not been studied in the literature. As mentioned earlier, Verdicchio et al. [16] showed that at $1200 \mathrm{~K}$, the alcohol elimination, which is equivalent to an isomerization in the case of cyclic structure, accounts for $9.8 \%$ of the total flux of unimolecular decomposition of THF. $\mathrm{H}_{2}$ elimination flux, ultimately yielding 2,3-dihydrofuran and $\mathrm{H}_{2}$ represents $9.1 \%$. Under these conditions (at low conversion), almost $20 \%$ of the unimolecular degradation of THF occurs through pericyclic reactions. The remaining flux of unimolecular decomposition involves diradicalar and carbene intermediates with a high level of complexity. In the Tran et al. [17] study on THP pyrolysis, a similar result was observed at low conversion: $9 \%$ of the total consumption flux of THP occurs through the alcohol elimination mechanism. Note that no $\mathrm{H}_{2-}$ elimination path was envisaged by these authors. The transition state structures associated with the 4centers rearrangement in cyclic ethers with no lateral alkyl group are bicyclic as the $\mathrm{H}$-atom transferred onto the $\mathrm{O}$-atom creates a bridge across positions 1 and 3 (bicyclo[2.1.1]ether). The presence of a lateral alkyl group in position 2, allows the 4-center pericyclic reaction to occur through fused rings created across the $\mathrm{C}-\mathrm{O}$ bond of the cyclic ether (bicyclo[1.2.0]ether). Figure 4 presents the potential energy 
surface (PES) computed for 2-MTHF pericyclic reactions. M1 is formed through a bicyclo[1.2.0]ether transition state, while M2 and M3 formation involves bicyclo[2.1.1]ether saddle points.

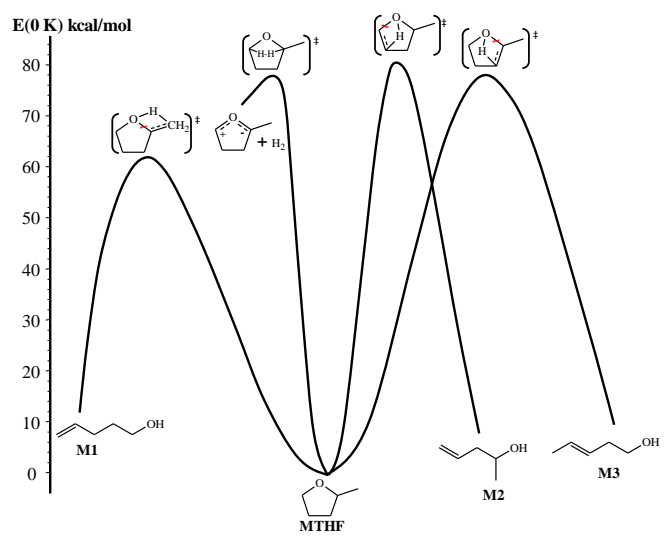

Figure 4: PES of 2-MTHF pericyclic reactions (G4 calculations at $0 \mathrm{~K}$ ).

As 2-MTHF is asymmetric, the 4-center rearrangement across the tetrahydrofuran ring can occur by the internal transfer of $\mathrm{H}$-atoms in position 3 or 4, leading to, respectively M3 and M2. By analogy with cylic alkyl ring openings [28], we shall name this kind of process endo as the double bond is formed inside the ether ring. Internal shift of $\mathrm{H}$-atom in position 3 is favored by $2.6 \mathrm{kcal} \mathrm{mol}^{-1}$ over the one in position $4\left(80.5 \mathrm{vs} 77.9 \mathrm{kcal} \mathrm{mol}^{-1}\right)$. This can be explain by the nature of the carbon atom involved in the $\mathrm{C}-\mathrm{O}$ bond where the $\mathrm{O}-\mathrm{C}_{\text {secondary }}$ bond (broken in 2-MTHF $\rightarrow \mathrm{M} 2$ ) is expected to be more difficult to break than the $\mathrm{O}-\mathrm{C}_{\text {tertiary }}$ bond $(2-\mathrm{MTHF} \rightarrow \mathrm{M} 3)$. For the THF system, Verdicchio et al. [16] reported a computed value of $80.4 \mathrm{kcal} \mathrm{mol}^{-1}$ at the CBS-QB3 level of theory. This is similar to our computed value for the reaction 2-MTHF $\rightarrow$ M2 which involves a similar molecular mechanism. To ensure the consistency of our results, we also performed theoretical calculations on the pericyclic reactions of THF, using several level of calculations. Results given in Table S1, shows that high level calculations predict an energy barrier of about $80 \mathrm{kcal} \mathrm{mol}^{-1}$ at $0 \mathrm{~K}$. These results confirm that the 4center alcohol formation involving a bicyclic[2.2.1] transition state in tetrahydrofuran derivatives involves a critical energy higher than that for acyclic ethers by about $13 \mathrm{kcal} \mathrm{mol}^{-1}$. 
The reaction 2-MTHF $\rightarrow$ M1 occurs through a bicyclic transition state where the transferred $\mathrm{H}$ atom comes from outside of the cyclic part of the molecule. We shall named this process an exo pericyclic rearrangement. This 4-center alcohol formation features a low energy barrier compared to the endo ones (2-MTHF $\rightarrow \mathrm{M} 2$, M3) with a computed value of $62.8 \mathrm{kcal} \mathrm{mol}^{-1}$. This activation energy is even lower by $4.3 \mathrm{kcal} \mathrm{mol}^{-1}$ compared to the similar elementary reaction in DEE. As far as we know, this is the first time that a study reports a lower activation energy for the 4-center alcohol formation in cyclic ethers compared to acyclic ethers. Note that this statement is only true for the exo pericyclic rearrangement. One can assume that the balance between the increase of ring strain to form the exo bicyclic transition state structure and the gain of ring strain energy released by the ring opening is favoring the latter contribution to the total energy barrier [29].

The $\mathrm{H}_{2}$-elimination from 2-MTHF faces an energy barrier of $78.2 \mathrm{kcal} \mathrm{mol}^{-1}$ which is competitive with the endo rearrangements. The value computed for THF at the same level of calculation (78.6 $\mathrm{kcal} \mathrm{mol}^{-1}$ ) is identical given the uncertainty associated with the $\mathrm{G} 4$ method. From a potential energy point of view, it can be seen that the $\mathrm{H}_{2}$ elimination is crucial if there is no exo 4-center pericyclic reactions available, i.e., it depends strongly on the molecular structure.

Similar calculations on the $\mathrm{H}_{2}$ elimination and the endo / exo 4-centers alcohol formation reactions were performed on 2,5-dimethyltetrahydrofuran (2,5-DMTHF), THP, 2-methyltetrahydropyran (2-MTHP), and 2,6-dimethyltetrahydropyran (2,6-DMTHP). Computational results are given in Table 2. 
Table 2: Pericyclic reactions of cyclic ethers: energy barriers and enthalpies of reaction of computed at the G4 level of theory $(0 \mathrm{~K})$.

\begin{tabular}{|c|c|c|}
\hline & Energy barrier (0 K) & $\Delta_{\mathrm{r}} \mathbf{H}(\mathbf{0} \mathbf{K})$ \\
\hline THF $\rightarrow$ 3-buten-1-ol (endo) & 79.8 & 6.7 \\
\hline $\mathrm{THF} \rightarrow \mathrm{C}_{4} \mathrm{H}_{6} \mathrm{O}+\mathrm{H}_{2}$ & 78.6 & 69.2 \\
\hline 2-MTHF $\rightarrow$ 4-penten-1-ol (exo) & 62.8 & 11.8 \\
\hline 2-MTHF $\rightarrow$ 4-penten-2-ol (endo, branched alcohol) & 80.5 & 7.7 \\
\hline 2-MTHF $\rightarrow$ 3-penten-1-ol (endo, linear alcohol) & 77.9 & 9.1 \\
\hline $2-\mathrm{MTHF} \rightarrow \mathrm{C}_{5} \mathrm{H}_{8} \mathrm{O}+\mathrm{H}_{2}$ & 78.2 & 69.1 \\
\hline 2,5-DMTHF $\rightarrow$ 5-hexen-2-ol (exo) & 64.6 & 13.2 \\
\hline 2,5-DMTHF $\rightarrow$ 4-hexen-2-ol (endo, linear alcohol) & 78.4 & 10.0 \\
\hline 2,5-DMTHF $\rightarrow \mathrm{C}_{6} \mathrm{H}_{10} \mathrm{O}+\mathrm{H}_{2}$ & 77.7 & 67.9 \\
\hline THP $\rightarrow$ 4-penten-1-ol (endo) & 77.3 & 11.0 \\
\hline $\mathrm{THP} \rightarrow \mathrm{C}_{5} \mathrm{H}_{8} \mathrm{O}+\mathrm{H}_{2}$ & 77.7 & 70.8 \\
\hline 2-MTHP $\rightarrow$ 5-hexen-1-ol (exo) & 66.9 & 16.0 \\
\hline 2-MTHP $\rightarrow$ 5-hexen-2-ol (endo, branched alcohol) & 77.3 & 12.5 \\
\hline 2-MTHP $\rightarrow$ 4-hexen-1-ol (endo, linear alcohol) & 76.0 & 13.6 \\
\hline 2-MTHP $\rightarrow \mathrm{C}_{6} \mathrm{H}_{10} \mathrm{O}+\mathrm{H}_{2}$ & 77.6 & 72.3 \\
\hline 2,6-DMTHP $\rightarrow$ 6-hepten-2-ol (exo) & 67.0 & 17.4 \\
\hline 2,6-DMTHP $\rightarrow$ 5-hepten-2-ol (endo) & 76.1 & 15.1 \\
\hline 2,6-DMTHP $\rightarrow \mathrm{C}_{7} \mathrm{H}_{12} \mathrm{O}+\mathrm{H} 2$ & 77.4 & 74.1 \\
\hline
\end{tabular}

It can be seen in Table 2, that the PES of 2,5-DMTHF is essentially the same as 2-MTHF. Tetrahydropyran derivatives are 6-membered rings and the evolution of the energy barriers compared to the case of tetrahydrofuran leads to interesting results. THP has no lateral alkyl group, and can decompose via the 4-centers alcohol formation reaction, which is endo by definition, or through the $\mathrm{H}_{2}$ elimination mechanism. THP 4-centers rearrangement faces an energy barrier of $77.3 \mathrm{kcal} \mathrm{mol}^{-1}$, which is $2.5 \mathrm{kcal} \mathrm{mol}^{-1}$ below the critical energy computed for the same process in THF. It partially reflects the difference of ring strain energy contributions to enthalpy between THP $\left(0.5 \mathrm{kcal} \mathrm{mol}^{-1}\right)$ and THF (5.9 $\mathrm{kcal} \mathrm{mol}^{-1}$ ) [17]. The addition of methyl groups on the pyran cycle (in position 2/5) allows, again, the exo 4-center alcohol elimination to occur. The computed energy barrier for this process in 2-MTHP (and 
2,6-DMTHP) is $66.9 \mathrm{kcal} \mathrm{mol}^{-1}$ which is mostly identical to the critical energy calculated for the same reaction in acyclic ethers. The powerful logic of a Benson approach is demonstrated here: as the ring strain of tetrahydropyran is almost zero, the estimation of the activation energy for this exo process would lead to use the same value as the one for DEE. One noteworthy fact is that the energy barriers of the exo 4-centers rearrangements of THP derivatives is higher than the ones for THF derivatives. It can be also noted that $\mathrm{H}_{2}$ elimination critical energies are almost independent from the size of the ether ring. In THP derivatives, the loss of $\mathrm{H}_{2}$ involves slightly lower values than in the case of THF.

Figure 5 depicts the computed high pressure limit rate constants for THF, 2-MTHF, THP and 2MTHP pericyclic reactions.

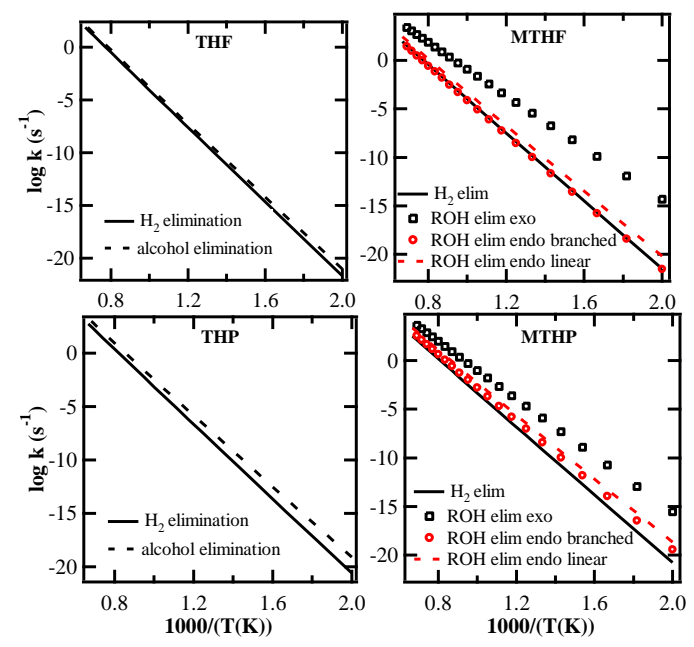

Figure 5: High pressure limit rate constants calculated for the pericyclic reactions in THF, 2-MTHF, THP and 2MTHP.

In the case of unalkylated rings, THF and THP, it appears that $\mathrm{H}_{2}$ eliminations and endo 4centers rearrangements are competitive. In order to compare the kinetics of pericyclic reactions in ethers, we calculated a purely pericyclic branching ratio which is defined as the ratio of a single pericyclic reaction rate constant over the sum of all possible pericyclic reaction rate constants. In the case of THF the computed branching ratios are $35 \%$ for $\mathrm{H}_{2}$ release and $65 \%$ for alcohol formation while these ratios are, respectively, $17 \%$ and $83 \%$ for THP at $1000 \mathrm{~K}$. Therefore, $\mathrm{H}_{2}$ elimination cannot be neglected in the thermal degradation of cyclic ethers when no exo 4-center concerted isomerization is 
available, e.g., for mono or poly cyclic rings with no lateral alkyl chain in position 2 and/or 5 . When a methyl group is located in $\beta$-position of the $\mathrm{O}$-atom pertaining to the ring, the exo 4-centers rearrangement becomes preponderant as illustrated by the rate constants for 2-MTHF and 2-MTHP in Figure 5. At $1000 \mathrm{~K}$, the branching ratio for the exo 4-centers rearrangement in 2-MTHF is dominant with a computed value of $99 \%$. For 2-MTHP, this process is still dominant with a branching ratio of $93 \%$ at $1000 \mathrm{~K}$ while the two endo 4-center alcohol formations accounts for $7 \%$. Similar plots were obtained for 2,5-DMTHF and 2,6-DMTHP and are given in Figure S1. It can be noted that the high pressure limit rate constants are generally higher in THF derivatives compared to THP derivatives. As an example, at $1000 \mathrm{~K}$, the $\mathrm{H}_{2}$ elimination is 8 times faster in THF compared to THP and the endo 4center rearrangement is 20 times faster.

Reaction rate rules for pericyclic reactions in cyclic ethers can be derived from these kinetic results. Comparisons of similar reactions processes (endo, exo 4-centers isomerization, $\mathrm{H}_{2}$ eliminations) between compounds featuring a similar reactive environment in the transition states structures show that such rate rules can be determined with confidence (Figure S2). The following parameters can be established to define the reaction rate rules: size of the cyclic ether (5- or 6- membered ring), position of the lateral alkyl group, nature of the H-atom(s) transferred. For any cyclic ether, endo 4-center isomerization and $\mathrm{H}_{2}$ elimination has to be included. If a lateral alkyl group is located in position 2/5 on the ether ring, then the exo 4-centers reaction has to be considered also. Table 3 gives the correlation established in this work to apply this rule in the development of chemical kinetic models.

$\mathrm{H}_{2}$ eliminations leads to the formation of a zwitterion that was shown to readily isomerize to 2,3dihydrofuran, in the case of THF [16], with a fitted activation energy of $18.9 \mathrm{kcal} \mathrm{mol}^{-1}$. In the case of THP, a similar isomerization reaction yielding 3,4-dihydropyran, was found in our study with a lower energy barrier of $9.2 \mathrm{kcal} \mathrm{mol}^{-1}$. We therefore recommend to use the corresponding dihydrofuran/pyran as the products of $\mathrm{H}_{2}$ eliminations. 
Table 3: Reaction rate rule for exo and endo alcohol formation and $\mathrm{H}_{2}$ elimination in cyclic ethers. $\mathrm{k}=\mathrm{A} * \mathrm{~T}^{\mathrm{n} *} \exp (-$ E/RT).

\begin{tabular}{|c|c|c|c|c|c|c|}
\hline \multirow{2}{*}{ H-type* } & \multicolumn{3}{|c|}{ xTHF } & \multicolumn{3}{|c|}{ xTHP } \\
\hline & $\mathrm{A}\left(\mathrm{s}^{-1}\right)$ & $\mathrm{n}$ & E (cal) & $\mathrm{A}\left(\mathrm{s}^{-1}\right)$ & $\mathrm{n}$ & E (cal) \\
\hline \multicolumn{7}{|c|}{ exo alcohol formation } \\
\hline $\mathrm{p}$ (x=Methyl) & $1.570 \times 10^{4}$ & 2.510 & 57859.9 & $1.778 \times 10^{6}$ & 2.197 & 63401.6 \\
\hline $\mathrm{s}$ ( $\mathrm{x}=$ Ethyl) & $2.412 \times 10^{5}$ & 2.203 & 58460.8 & $1.268 \times 10^{6}$ & 2.253 & 68906.6 \\
\hline$t(x=$ isoPropyl $)$ & $1.277 \times 10^{6}$ & 1.960 & 61154.2 & $4.064 \times 10^{6}$ & 2.088 & 69702.4 \\
\hline \multicolumn{7}{|c|}{ endo- 1 alcohol formation } \\
\hline \multicolumn{7}{|c|}{ endo-2 alcohol formation } \\
\hline & $2.339 \times 10^{9}$ & 1.368 & 75711.0 & $2.519 \times 10^{7}$ & 2.052 & 71988.0 \\
\hline \multicolumn{7}{|l|}{$\mathrm{H}_{2}$ elimination } \\
\hline & $3.473 \times 10^{10}$ & 0.956 & 79170.0 & $4.785 \times 10^{11}$ & 0.748 & 78427.0 \\
\hline
\end{tabular}

\section{Concluding remarks}

For decades, alcohol elimination in acyclic ethers thermal decomposition have been known to be preponderant. On the other hand, the role of this pericyclic mechanism has almost not been studied for cyclic ethers. In this study we explored in details the concerted alcohol formation pathways for acyclic and cyclic ethers. A new unimolecular decomposition route involving a concerted $\mathrm{H}_{2}$ elimination was also envisaged for all the ethers studied. This concerted process was found to be negligible in acyclic ether where the alcohol elimination is preponderant for temperature below about $1200 \mathrm{~K}$. Above $1200 \mathrm{~K}$, initial $\mathrm{C}-\mathrm{O}$ bond fissions become competitive. The role of concerted eliminations in the thermal decomposition of cyclic ethers is more complex. It was shown that for 5- and 6-member ether rings, $\mathrm{H}_{2}$ eliminations leading to the formation of dihydrofuran / pyran and endo 4-centers rearrangements, yielding an unsaturated alcohol, are almost equivalent in terms of branching ratios if no lateral alkyl group is located in position $2 / 5$. The presence of a lateral alkyl group allows an exo concerted alcohol formation which is predicted to be dominant over the others unimolecular pericyclic reactions. Based on the work of Verdicchio et al. [16] on THF, where diradical and carbene unimolecular initiations were also considered, the concerted pathways are expected to contribute to about $20 \%$ of the total 
unimolecular consumption flux of the fuel at low conversion. The same conclusion should stand for THP. If cyclic ethers with, at least, one lateral alkyl group in position 2 were considered, the exo rearrangement into an unsaturated alcohol is expected to be dominant given the low energy barrier of this process compared to the critical energies of competitive diradical and carbene unimolecular

initiations $\left(\approx 80 \mathrm{kcal} \mathrm{mol}^{-1}\right)$. Reaction rate rules were proposed to determine the kinetic parameters of concerted reactions involved in the combustion and pyrolysis models of ethers.

\section{Acknowledgements}

This work was granted access to the HPC resources of CINES under the allocation 2015087249 made by GENCI. 
References

[1] G.W. Huber, S. Iborra, A. Corma, Chemical reviews 106 (2006) 4044-4098.

[2] L.S. Tran, B. Sirjean, P.-A. Glaude, R. Fournet, et al., Energy 43 (2012) 4-18.

[3] L. Cai, A. Sudholt, D.J. Lee, F.N. Egolfopoulos, et al., Combustion and Flame 161 (2014) 798809.

[4] S. Di Tommaso, P. Rotureau, B. Sirjean, R. Fournet, et al., Process Safety Progress 33 (2014) 64-69.

[5] U. Burke, K.P. Somers, P. O'Toole, C.M. Zinner, et al., Combustion and Flame 162 (2015) 315330.

[6] K. Yasunaga, J.M. Simmie, H.J. Curran, T. Koike, et al., Combustion and Flame 158 (2011) 1032-1036.

[7] C. Danby, G. Freeman, in: Proceedings of the Royal Society of London A: Mathematical, Physical and Engineering Sciences, The Royal Society, 1958, pp. 40-48.

[8] K. Laidler, D. McKenney, Proceedings of the Royal Society of London. Series A, Mathematical and Physical Sciences (1964) 505-516.

[9] J. Foucaut, R. Martin, Journal de chimie physique et de physico-chimie biologique 75 (1978)

132-144.

[10] K. Yasunaga, Y. Kuraguchi, Y. Hidaka, O. Takahashi, et al., Chemical Physics Letters 451 (2008) 192-197.

[11] K. Yasunaga, F. Gillespie, J. Simmie, H. Curran, et al., The Journal of Physical Chemistry A 114 (2010) 9098-9109.

[12] M.J. Al Rashidi, A.C. Davis, S.M. Sarathy, Proceedings of the Combustion Institute 35 (2015) 385-392.

[13] K.S. Pitzer, W.D. Gwinn, The Journal of Chemical Physics 10 (1942) 428-440.

[14] B. Sirjean, R. Fournet, Physical Chemistry Chemical Physics 15 (2013) 596-611.

[15] K. Moshammer, S. Vranckx, H.K. Chakravarty, P. Parab, et al., Combustion and Flame 160 (2013) 2729-2743.

[16] M. Verdicchio, B. Sirjean, L.S. Tran, P.-A. Glaude, et al., Proceedings of the Combustion Institute 35 (2015) 533-541.

[17] L.-S. Tran, R. De Bruycker, H.-H. Carstensen, P.-A. Glaude, et al., Combustion and Flame 162 (2015) 4283-4303.

[18] N.J. Labbe, V. Seshadri, T. Kasper, N. Hansen, et al., Proceedings of the Combustion Institute 34 (2013) 259-267.

[19] P. Dagaut, M. McGuinness, J. Simmie, M. Cathonnet, Combustion science and technology 129 (1997) 1-16.

[20] I. Alecu, J. Zheng, Y. Zhao, D.G. Truhlar, Journal of chemical theory and computation 6 (2010) 2872-2887.

[21] J.C. Lizardo-Huerta, B. Sirjean, R. Bounaceur, R. Fournet, Physical Chemistry Chemical Physics Submitted (2015).

[22] P. Vansteenkiste, D. Van Neck, V. Van Speybroeck, M. Waroquier, The Journal of Chemical Physics 124 (2006) 044314.

[23] C. Eckart, Physical Review 35 (1930) 1303-1309.

[24] R. Sivaramakrishnan, J. Michael, A. Wagner, R. Dawes, et al., Combustion and Flame 158 (2011) 618-632.

[25] Y. Georgievskii, J.A. Miller, M.P. Burke, S.J. Klippenstein, The Journal of Physical Chemistry A 117 (2013) 12146-12154.

[26] Y. Georgievskii, A. Jasper, J. Zador, M. Burke, et al., Unpublished (2014). 
[27] I. Seres, P. Huhn, International journal of chemical kinetics 18 (1986) 829-836.

[28] B. Sirjean, P.A. Glaude, M.F. Ruiz-Lopez, R. Fournet, Journal of Physical Chemistry A 112 (2008) 11598-11610.

[29] B. Sirjean, P.A. Glaude, M.F. Ruiz-Lopez, R. Fournet, Journal of Physical Chemistry A 110 (2006) 12693-12704.

\section{Supplemental material:}

Table S1: Comparisons of different levels of calculations for pericyclic reactions in DEE and THF

Table S2: Parameters used in the master equation simulation of the $\mathrm{H}_{2}$ elimination in DME.

Figure S1: High-pressure limit rate constants calculated for the pericyclic reactions in 2,5-DMTHF and 2,6-DMTHP.

Figure S2: Comparisons of high-pressure limit rate constants for similar pericyclic reactions in different cyclic ethers. 